Editorial

\title{
Prosthesis: New Technological Opportunities and Innovative Biomedical Devices
}

\author{
Marco Cicciù (iD) \\ Department of Biomedical and Dental Sciences, Morphological and Functional Images, School of Dentistry, \\ University of Messina, Policlinico G. Martino, Via Consolare Valeria, 98100 Messina, Italy; mcicciu@unime.it
}

Received: 4 June 2019; Accepted: 17 June 2019; Published: 18 June 2019

\begin{abstract}
The objective of this editorial is to present Prosthesis as the main reference point for all researchers involved in biomedical device and prosthesis investigations. Material science and the development of new technologies in the field of regenerative medicine signaled a meeting point between medicine and engineering towards the next generation of prosthetic devices. The aim of the journal is to favor studies and the diffusion of new technologies in the field of rehabilitation medicine. Journal topics will cover everything related to the rehabilitation in humans or animals through the application of new device as well as new advanced surgical techniques and materials.
\end{abstract}

Keywords: prosthesis; biomedicine; regenerative medicine

"Sharing knowledge, mistakes, and experiences is the basis of progress."

The production of customized prosthetic devices has seen a large increase in the last few years. The constant advancement of research and science aimed at the patient's quality of life improvements has allowed fundamental technological progress. The last ten years signaled the beginning of a new era in the field of regenerative medicine. Today, a multidisciplinary approach and close cooperation between medical doctors and bioengineers are common practices for the management of prosthesis implantation in the human body.

"Prosthesis" is a simple word, closely related to human progress and development. Its Greek origin and etymology, "prosthesis", "prò-theis", and "protithèmi", mean "to position forward" or "to position in the place of ....". The history of the word is well known from ancient Egyptian and Hellenic cultures, and it is related to military medicine and the first war in which doctors or surgeons tried to replace arms, hands, or portions of the body lost in battle or conflict, or from degenerative diseases [1,2].

History informs us how for every problem, humans look for a solution; the Egyptians were the first pioneers of prosthetic technology, dating from c. 2750-2625 AD. Their rudimentary prosthetic limbs were made of fiber and are believed to have been worn more for aesthetic and non-functional purposes. However, scientists have recently discovered what is said to be the world's first prosthetic finger, installed on an Egyptian mummy. This prosthesis would seem to play a functional as well as aesthetic role. Another historical moment related to the evolution of the prosthesis was documented by Plino il Vecchio (23-79 AD). He wrote about a Roman general who fought in the second Punic War (218-210 BC) with an amputated right arm. This soldier had an iron hand, which allowed him to hold his shield, and thus he was able to return to fight $[3,4]$.

The American Civil War created a high number of amputations, forcing Americans to enter the field of prosthetics as government policy. James Hanger, one of the amputees before the Civil War, developed what was later patented as "Hanger Limb". People like Hanger, Selpho, Palmer, and A. A. Marchi helped transform and advance the prosthetic field with their refinements in the mechanisms and materials of the devices. Unlike the civil war, the First World War did not favor the advancement of prosthetic development. Despite the lack of technological advances, the army 
surgeon understood the importance of the discussion of technology and prosthetic development, which eventually led to the formation of the American Orthotic and Prosthetic Association (AOPA). Following the Second World War, veterans were not satisfied with the lack of technology in their devices and called for an improvement, so the US government negotiated an agreement with military companies to improve prosthesis function and aesthetics rather than produce weapons. This agreement paved the way for the development and production of modern prostheses.

Today prosthesis can be classified as internal or external in connection to the body area to be treated. Modern prosthetic medical devices are much lighter, made of plastic, aluminum or titanium, and composite materials to provide amputee patients with more functional devices. Aesthetics and function are two parameters that cannot be separated in the production of prostheses. Therefore, the study of human load during walking and evaluation of the elastic modulus of each body tissue increased the integration of prosthetic devices, decreasing their wear. In addition to being lighter, the new devices are modeled on the patient with the advent of microprocessors, computer chips, and robotics. Today, prostheses are designed to return amputees to the lifestyle they were used to, rather than only providing basic functionality or simply a more pleasing appearance. Current prostheses are more realistic, have silicone covers, and are able to mimic the function of a natural limb now more than any time before.

Nowadays, advanced application in the field of Engineering is signaling the final integration between regenerative medicine and mechanical computerized assisted engineering [5].

Reverse engineering, design software, and three-dimensional imaging diagnostic systems, exploited together, now allow patient-specific, predictable, long-term rehabilitation. Custom-made and personalized prostheses not only include factors of aesthetic and ergonomic aspects, but also parameters related to the biomechanical nature, which most clinicians are concerned about in the intervention of long- or short-term side effects. Therefore, the procedures of finite element analysis, and the Von Mises tests currently allow one to virtualize a rehabilitation before it is realized and consider the mechanical stresses under load even before the prosthesis is created. The other aspect of rehabilitation is closely related to the phases of realization that now provide a personalized precision realization with 3D printing techniques or three-dimensional milling machines.

As scientists and researchers, we should be honored to be part of this technological time, and Prosthesis, as a biomedical journal, can be the main instrument for sharing knowledge and comparing experience pointed towards human benefit and progress.

Prosthesis (ISSN 2673-1592) is an international peer-reviewed open access journal on rehabilitation medicine published quarterly online by MDPI.

Conflicts of Interest: The author declares no conflict of interest.

\section{References}

1. Priorov, N.N. Amputation of the Extremities and Prosthesis. Br. Med. J. 1945, 1, 178-179. [CrossRef] [PubMed]

2. Gillis, L. Recent advances in the treatment of arm amputations, kineplastic surgery and arm prostheses. Ann. R. Coll. Surg. Engl. 1948, 3, 227-247. [PubMed]

3. Mowlem, R. Surgery and prostheses. Procl. R. Soc. Med. 1950, 43, 711-716.

4. Wang, T.; Liu, N.; Su, Z.; Li, C. A New Time-Frequency Feature Extraction Method for Action Detection on Artificial Knee by Fractional Fourier Transform. Micromachines 2019, 10, 333. [CrossRef] [PubMed]

5. Cicciù, M.; Cervino, G.; Milone, D.; Risitano, G. FEM Investigation of the Stress Distribution over Mandibular Bone Due to Screwed Overdenture Positioned on Dental Implants. Materials 2018, 11, 1512. [CrossRef] [PubMed] 